\title{
ON SUPERCOMPLETE SPACES. III
}

\author{
AARNO HOHTI
}

\begin{abstract}
We show that for each positive integer $n$ there is a fine uniform space $X$, topologically a subspace of the real line, such that $X^{n}$ is supercomplete, but $X^{n+1}$ is not supercomplete. The space $X$ can also be chosen so that that $X^{n}$ is supercomplete for all $n \in N$, but the countably infinite power $X^{N}$ is not supercomplete.
\end{abstract}

1. Introduction. Our paper pertains to recent results of $M$. Hušek and J. Pelant on products of uniform spaces. In [6] J. Isbell proved that the locally fine coreflection of any product of fine completely metrizable separable spaces is fine. In the context of extending functions defined on certain subspaces of Cartesian products, Hušek and Pelant [3] essentially extended Isbell's result by showing that the locally fine coreflection of any product of fine Čech-complete paracompact spaces is fine. Their results include similar results for $(C$-) scattered paracompact spaces, extending the finite case considered in [2]. However, an interesting problem remains: Is the locally fine coreflection determined by its action on finite subproducts in case the factors are supercomplete or at least separable metrizable? in other words, given a supercomplete space $X$ with supercomplete finite powers, does it follow that an infinite power of $X$ is supercomplete? In this paper we shall see that the answer is negative.

We refer the reader to [6] or to the forthcoming book [4] for information on uniform spaces. For a uniform space $u X$, the locally fine coreflection [1] of $u X$ is denoted by $\lambda u X$. If $X$ is a uniformizable (i.e., completely regular) space, then we denote the corresponding fine uniform space by $\mathscr{F} X$. A uniform space $u X$ is called supercomplete if the hyperspace $H(u X)$ of all closed subsets of $X$ is complete. By a theorem of Isbell [5], $u X$ is supercomplete iff $X$ is topologically paracompact and $\lambda u X=\mathscr{F} X$. For any set $X$ let $\pi_{n}: X^{m} \rightarrow X$ denote the $n$th projection, where $m \geqslant n$.

2. Auxiliary results. Our result essentially depends on the notion of $n$-cardinality introduced by T. Przymusiński in [7]. Let $X$ be a set and let $A \subset X^{n}$, where $n \in N=\{1,2,3, \ldots\}$. Then the $n$-cardinality $|A|_{n}$ of $A$ is defined as the minimum of all cardinals $|Y|$, where $Y \subset X$ and

$$
A \subset\left(Y \times X^{n-1}\right) \cup\left(X \times Y \times X^{n-2}\right) \cup \cdots \cup\left(X^{n-1} \times Y\right) ;
$$

Received by the editors May 7, 1984 and, in revised form, April 11, 1985

1980 Mathematics Subject Classification. Primary 54E15.

Key words and phrases. Supercomplete, locally fine coreflection, fine space, $n$-cardinality. 
i.e., $A$ can be "killed" by the set of all codimension 1 hyperplanes going through the elements of $Y$. The following result is due to Van Douwen (the closed case) and Przymusińnki (the analytic case):

2.1. Theorem. Let $X$ be a Polish space, let $n \in N$, and let $A$ be an analytic subset of $X^{n}$. Then $|A|_{n}>\omega$ implies $|A|_{n}=2^{\omega}$.

We shall use 2.1 to construct a suitable subset of the real line $R$. Next we state a crucial lemma on supercompleteness of products of separable metrizable spaces. The proof is similar to the proof of a corresponding result given in [2].

2.2. Lemma. Let $Y$ be a dense subspace of a Polish space $X$, and let $n>1$. Then $\lambda(\mathscr{F} Y)^{n}=\mathscr{F}\left(Y^{n}\right)$ iff for each closed $K \subset X^{n} \sim Y^{n}$ there exists a $G_{\delta}$-set $G \subset X$ such that $Y \subset G$ and $G^{n} \cap K=\varnothing$.

3. The results. To prove our theorems we need the following lemma.

3.1. Lemma. Let $X$ be a Polish space, let $n>1$, and let $Y$ be a subspace of $X$ such that for $1 \leqslant k \leqslant n$, every closed subset of $X^{k} \sim Y^{k}$ has countable $k$-cardinality. Then $\lambda(\mathscr{F} Y)^{n}=\mathscr{F}\left(Y^{n}\right)$.

Proof. By 2.2 we must show that for each closed set $K \subset X^{n} \sim Y^{n}$ there is a $G_{\delta}$-set $G \subset X$ such that $Y \subset G$ and $G^{n} \cap K=\varnothing$. Thus, it will be enough to find a countable set $D \subset X \sim Y$ such that

$$
K \subset\left(D \times X^{n-1}\right) \cup\left(X \times D \times X^{n-2}\right) \cup \cdots \cup\left(X^{n-1} \times D\right) .
$$

Then we can take $G=X \sim D$. We proceed by induction on $n$. Therefore, consider the following statement: If $X, Y$ are as in 3.1, then for each closed $K \subset X^{m} \sim Y^{m}$ there is a countable $D \subset X \sim Y$ such that $K \subset\left(D \times X^{m-1}\right) \cup \cdots \cup\left(X^{m-1} \times D\right)$. The claim is trivially true for $m=1$. In this case we simply take $D=K$. Suppose that we have proved the statement for $1 \leqslant m \leqslant k \leqslant n$. Let $K \subset X^{k+1} \sim Y^{k+1}$ be closed. By the assumption, $|K|_{k+1} \leqslant \omega$, and hence there is a countable set $D^{\prime} \subset X$ such that $K \subset\left(D^{\prime} \times X^{k}\right) \cup \cdots \cup\left(X^{k} \times D^{\prime}\right)$. For each point $p$ of $D^{\prime} \cap Y$, put $K_{p, i}=K \cap \pi_{i}^{-1}(p)$, where $1 \leqslant i \leqslant k+1$, and let $K_{p, i}^{\prime}$ be the projection of $K_{p, i}$ into $X^{k}$ obtained by deleting the $i$ th coordinate. Then $K_{p, i}^{\prime} \subset X^{k} \sim Y^{k}$, for otherwise we would have $K \cap Y^{k+1} \neq \varnothing$. By the inductive hypothesis there is a countable set $D_{p, i} \subset X \sim Y$ such that $K_{p, i}^{\prime} \subset\left(D_{p, i} \times X^{k-1}\right) \cup \cdots \cup\left(X^{k-1} \times D_{p, i}\right)$. Put

$$
D=\cup\left\{D_{p, i}: p \in D^{\prime} \cap Y, 1 \leqslant i \leqslant k+1\right\} \cup\left(D^{\prime} \sim Y\right) .
$$

Then $D$ establishes the truth of our claim for $m=k+1$. This completes the proof.

3.2. THEOREM. For each $n \in N$ there is a subspace $X$ of $R$ such that $\lambda(\mathscr{F} X)^{n}=$ $\mathscr{F}\left(X^{n}\right)$, but $\lambda(\mathscr{F} X)^{n+1} \neq \mathscr{F}\left(X^{n+1}\right)$. Thus, $(\mathscr{F} X)^{n}$ is supercomplete, but $(\mathscr{F} X)^{n+1}$ is not supercomplete.

Proof. We construct the space $X$ by induction. First, let $a>0$, and let $\bar{a}=$ $\left(2 a, 4 a, \ldots, 2^{k} a, \ldots, 2^{n} a\right) \in R^{n}$. We define a map $\varphi: R^{n+1} \rightarrow R^{n}$ by setting

$$
\varphi\left(x_{1}, x_{2}, \ldots, x_{n+1}\right)=\left(x_{1}+x_{2}, x_{1}+x_{3}, \ldots, x_{1}+x_{n+1}\right) .
$$


For $1 \leqslant i \leqslant n$, let $\left\langle F_{\alpha}^{(i)}: \alpha<2^{\omega}\right\rangle$ be an enumeration of all closed subsets of $R^{i}$ of uncountable $i$-cardinality. By $2.1,\left|F_{\alpha}^{(i)}\right|=2^{\omega}$ for all $\alpha<2^{\omega}$. Now let $A$ denote the set of all integer multiples of $a$. For each $\alpha<2^{\omega}$, put

$$
\tilde{F}_{\alpha}^{(i)}=F_{\alpha}^{(i)} \sim \bigcup\left\{\pi_{k}^{-1}[A]: 1 \leqslant k \leqslant i\right\} .
$$

Thus, no point of $\tilde{F}_{\alpha}^{(i)}$ has a coordinate which is an integer multiple of $a$.

In the construction of $X$ the first step is similar to the subsequent ones; hence we omit it. Thus, assume that $\alpha<2^{\omega}$ and that we have constructed the sets $X_{\beta} \subset R$ for all $\beta<\alpha$ with the following properties:

(1) $X_{\beta}^{i} \cap \tilde{F}_{\beta}^{(i)} \neq \varnothing$ whenever $\beta<\alpha$ and $1 \leqslant i \leqslant n$;

(2) $\left|X_{\beta}\right|<2^{\omega}$;

(3) $\bar{a} \in R^{n} \sim \varphi\left[X_{\beta}^{n+1}\right]$ and

(4) $X_{\beta} \subset X_{\beta^{\prime}}$ whenever $\beta<\beta^{\prime}<\alpha$.

We define a subset $E_{\alpha}$ of $R$ by setting

$$
E_{\alpha}=\left\{x \in R: x+y=2^{i} a \text { for some } y \in X_{\beta} \text {, where } \beta<\alpha, 0 \leqslant i \leqslant n-1\right\} .
$$

Then $\left|E_{\alpha}\right|<2^{\omega}$. As the $n$-cardinality of $\tilde{F}_{\alpha}^{(n)}$ is $2^{\omega}$, we can find a point

$$
\left(p_{1}, \ldots, p_{n}\right) \in \tilde{F}_{\alpha}^{(n)} \sim \bigcup\left\{\pi_{k}^{-1}\left[E_{\alpha}\right]: 1 \leqslant k \leqslant n\right\} .
$$

Put $X_{\alpha}^{\prime}=\bigcup\left\{X_{\beta}: \beta<\alpha\right\} \cup\left\{p_{1}, \ldots, p_{n}\right\}$. Suppose that $\left(z_{1}, \ldots, z_{n+1}\right) \in\left(X_{\alpha}^{\prime}\right)^{n+1}$ and that $\varphi\left(z_{1}, \ldots, z_{n+1}\right)=\bar{a}$. Then $\left\{z_{1}, \ldots, z_{n+1}\right\} \cap X_{\beta}=\varnothing$ for all $\beta<\alpha$, by the assumption that $\bar{a} \notin \bigcup\left\{\varphi\left[X_{\beta}^{n+1}\right]: \beta<\alpha\right\}$ and the way $\left(p_{1}, \ldots, p_{n}\right)$ was chosen. Hence, $\left\{z_{1}, \ldots, z_{n+1}\right\} \subseteq\left\{p_{1}, \ldots, p_{n}\right\}$. There exist $i, j, 1 \leqslant i<j \leqslant n+1$ and a $k$ such that $z_{i}=z_{j}=p_{k}$. We must have $i=1$, for otherwise $2^{i-1} a=z_{1}+z_{i}=$ $z_{1}+z_{j}=2^{j-1} a$. Hence, $z_{1}+z_{j}=2^{j-1} a$ implies that $p_{k}=2^{j-2} a$ (and thus $z_{i}=$ $\left(2^{i-1}-2^{j-2}\right) a$ for $\left.2 \leqslant i \leqslant n\right)$, which contradicts the assumption that in the coordinates of $\tilde{F}^{(n)}$ there are no integer multiples of $a$. Hence, $\varphi\left[\left(X_{\alpha}^{\prime}\right)^{n+1}\right] \subset R^{n} \sim\{\bar{a}\}$. Put

$$
E_{\alpha}^{\prime}=\left\{x \in R: x+y=2^{i} a \text { for some } y \in X_{\alpha}^{\prime} \text {, where } 0 \leqslant i \leqslant n-1\right\} .
$$

As before, we can find a point

$$
\left(p_{1}^{\prime}, \ldots, p_{n-1}^{\prime}\right) \in \tilde{F}_{\alpha}^{(n-1)} \sim \bigcup\left\{\pi_{k}^{-1}\left[E_{\alpha}^{\prime}\right]: 1 \leqslant k \leqslant n-1\right\} .
$$

Define $X_{\alpha}^{\prime \prime}=X_{\alpha}^{\prime} \cup\left\{p_{1}^{\prime}, \ldots, p_{n-1}^{\prime}\right\}$. Then the argument used above shows that $\varphi\left[\left(X_{\alpha}^{\prime \prime}\right)^{n+1}\right] \subset R^{n} \sim\{\bar{a}\}$. Proceeding in this way downward (we wanted to start from the nontrivial end), we obtain a set $X_{\alpha}$ such that $\varphi\left[X_{\alpha}^{n+1}\right] \subset R^{n} \sim\{\bar{a}\}$, $\left|X_{\alpha}^{n+1}\right|<2^{\omega}$ and $X_{\alpha}^{i} \cap F_{\alpha}^{(i)} \neq \varnothing$ whenever $1 \leqslant i \leqslant n$. This completes the inductive definition of the sets $X_{\alpha}$. Put $X=\bigcup\left\{X_{\alpha}: \alpha<2^{\omega}\right\}$.

By 3.1, we have $\lambda(\mathscr{F} X)^{n}=(\mathscr{F} X)^{n}$. Indeed, if $K$ is any closed subset of $R^{m} \sim X^{m}, 1 \leqslant m \leqslant n$, then $|K|_{m}>\omega$ would imply $K=F_{\alpha}^{(m)}$ for some $\alpha<2^{\omega}$, and consequently $K \cap X^{m} \neq \varnothing$, which is impossible.

To show that $\lambda(\mathscr{F} X)^{n+1} \neq \mathscr{F}\left(X^{n+1}\right)$, let $K=\left\{x \in R^{n+1}: \varphi(x)=\bar{a}\right\}$. Since $\varphi$ is continuous, $K$ is closed. Moreover, $\bar{a} \notin \varphi\left[X^{n+1}\right]$ implies $K \subset R^{n+1} \sim X^{n+1}$. If $\lambda(\mathscr{F} X)^{n+1}$ were fine, then there would exist a $G_{\delta}$-set $G \subset R$ such that $X \subset G$ and $G^{n} \cap K=\varnothing$. Since $X$ is dense in $R$, there would exist a first category set 
$Z \subset R \sim X$ such that

$$
K \subset\left(Z \times R^{n}\right) \cup\left(R \times Z \times R^{n-1}\right) \cup \cdots \cup\left(R^{n} \times Z\right) .
$$

However, this is impossible. This is readily seen by observing that $K=\{(x, 2 a-$ $\left.\left.x, 4 a-x, \ldots, 2^{n} a-x\right): x \in R\right\}$.

3.3. THEOREM. There is a subspace $X$ of $R$ such that $\lambda(\mathscr{F} X)^{n}=\mathscr{F}\left(X^{n}\right)$ for all $n \in N$, but $\lambda(\mathscr{F} X)^{N} \neq \mathscr{F}\left(X^{N}\right)$.

Proof. Now we consider the map $\varphi: R^{N} \rightarrow R^{N}$ defined by $\varphi\left(x_{1}, x_{2}, \ldots\right)=\left(x_{1}+\right.$ $\left.x_{2}, x_{1}+x_{3}, \ldots\right)$ and a point $\bar{a}=(2 a, 4 a, 8 a, \ldots)$ of $R^{N}$. Except for trivial changes the details are the same as those used to prove 3.2.

3.4. Corollary. For each $n \in N$ there is a fine separable metrizable space $X$ such that $X^{n}$ is supercomplete, but $X^{n+1}$ is not supercomplete. Also there is a fine separable metrizable space $X$ such that each finite power of $X$ is supercomplete, but $X^{N}$ is not supercomplete.

ACKNOWLEDgement. The author is grateful to Heikki Junnila for pointing out the relevance of the notion of $n$-cardinality and Bernstein subsets of $R$.

\section{REFERENCES}

1. S. Ginsberg and J. Isbell, Some operators on uniform spaces, Trans. Amer. Math. Soc. 93 (1959), 145-168.

2. A. Hohti, On supercomplete spaces. II (preprint).

3. M. Hušek and J. Pelant, Products and uniform spaces (manuscript).

4. M. Hušek and M. Rice, Uniform spaces (manuscript).

5. J. Isbell, Supercomplete spaces, Pacific J. Math. 12 (1962), 287-290.

6. Uniform spaces, Math. Surveys, no. 12, Amer. Math. Soc., Providence, R. I., 1964.

7. T. Przymusinski, On the notion of n-cardinality, Proc. Amer. Math. Soc. 69 (1978), 333-338.

Department of Mathematics, University of Helsinki, Hallituskatu 15, SF - 00100 Helsinki 10 , FINLAND 